\title{
Validation of Pre-operative Templating for Total Disc Replacement Surgery
}

\author{
JUSTIN F.M. HOLLENBECK, MS, ${ }^{1}$ JILL A. FATTOR, MS, MS-PA, ${ }^{2}$ VIKAS PATEL, BSME, MA, MD ${ }^{2}$ \\ EVALINA BURGER, MBCHB, MMED(ORTHO) ${ }^{2}$ PAUL J. RULLKOETTER, PHD, ${ }^{1}$ CHRISTOPHER M.J. \\ CAIN, MBBS, MD ${ }^{2}$ \\ ${ }^{1}$ Center for Orthopaedic Biomechanics, University of Denver, Denver, Colorado, ${ }^{2}$ Department of Orthopedics, University of Colorado, Denver, Aurora, Colorado
}

\begin{abstract}
Objectives: This was an analytic retrospective observational study. The aims were (1) to validate patient-specific templating process by comparing postoperative range of motion (ROM) with that predicted by the model, (2) to retrospectively determine the ideal implant size, height, configuration, and location to evaluate if the ROM achieved could have been improved, and (3) to correlate postoperative ROM and clinical outcome.

Background: Previous research revealed that after total disc replacement surgery, $34 \%$ of patients with less than $5^{\circ}$ of postoperative ROM developed adjacent segment disease. The match between patient anatomy (size, facet orientation, disc height) and implant parameters are likely to affect postoperative ROM and clinical outcomes.

Methods: Seventeen consecutive patients were implanted with 22 ProDisc-L devices between 2008 and 2015. Three-dimensional finite element (FE) models of the implanted segment were constructed from preoperative computed tomography scans and virtually implanted with the ProDisc-L implant. ROM was determined with the endpoints of facet impingement in flexion and implant contact in extension. FE templating was used to determine the optimal implant size and location. ROM was then measured directly from flexion and extension radiographs and compared to predicted ROM. Pre and postoperative Oswestry Disability Index (ODI) data were used to correlate ROM with clinical outcomes.

Results: No significant difference was found between the actual and predicted ROM. The computational templating procedure identified an optimal ROM that was significantly greater than actual ROM. The ROM in our cohort could have been improved by an average of $1.2^{\circ}$ or $12 \%$ had a different implant size or position been used.

Conclusions: FE analyses accurately predicted ROM in this cohort and can facilitate selection of the optimal implant size and location that we believe will increase the chance of achieving clinical success with the application of this technology.
\end{abstract}

TDR

Keywords: low back pain, adjacent segment disease, total disc replacement, range of motion, finite element modeling

\section{INTRODUCTION}

From 2000 to 2010, surgical treatment for lumbar degenerative disc disease has increased almost 3-fold in the United States. ${ }^{1}$ While clinical success rates of lumbar fusion treatment have been reported to exceed $80 \%,{ }^{2}$ this procedure also eliminates motion and, in cases with posterior instrumented fusion, has been associated with accelerated adjacent segment disease (ASD). ${ }^{3}$ Total disc replacement (TDR), introduced as an alternative treatment for this pathology, was hoped to minimize, if not eliminate symptomatic degeneration while preserving motion and reducing the incidence of ASD. ${ }^{4-9}$

Studies have reported that patients implanted with a TDR not only achieved significantly greater postoperative range of motion (ROM) but also maintained natural disc height more consistently, and experienced lesser device-related complications, lower pain scores, and greater clinical success rates than patients who underwent fusion surgeries. ${ }^{4-9}$ Further, Harrop et $\mathrm{al}^{8}$ reported a lower prevalence of ASD in TDR patients compared to fusion patients. Huang et $\mathrm{al}^{9}$ evaluated the ROM achieved and the incidence of ASD and found clear relationships between TDR motion and the presence of ASD at an average of 8.7-year follow-up where patients who achieved at least $5^{\circ}$ postoperative motion had $0 \%$ prevalence of ASD, while patients with motion less than $5^{\circ}$ had a $34 \%$ prevalence of ASD. In light of these findings, a 
Table 1. Age, weight, height, and body mass index (BMI) statistics differentiated by gender for 22 implanted levels mean \pm standard deviation (range).

\begin{tabular}{lrcccc}
\hline & N & Age & Weight (lb) & Height (in) & BMI \\
\hline Males & 16 & $36.3 \pm 6.7(24-50)$ & $190.6 \pm 37.5(147-257)$ & $69.4 \pm 3.1(65-74)$ & $27.6 \pm 3.4(23-33)$ \\
Females & 6 & $34.5 \pm 6.9(21-40)$ & $173.0 \pm 62.0(125-280)$ & $65.6 \pm 3.1(63-69)$ & $27.7 \pm 7.6(22-41)$ \\
Total & 22 & $35.8 \pm 6.6(21-50)$ & $185.9 \pm 44.0(125-280)$ & $68.3 \pm 3.5(63-74)$ & $27.6 \pm 4.6(22-41)$ \\
\hline
\end{tabular}

surgical goal is to select and place the implant in a position that will achieve at least $5^{\circ} \mathrm{ROM}$.

As the range of implants and their geometries are limited, key anatomical variables such as vertebra size, disc height, and facet orientation influence the compatibility of any particular implant with a patient who is under consideration for TDR treatment. Clinicians utilize preoperative radiographic overlay templates, intraoperative radiographs, and implant trials to choose an implant that maximizes footprint coverage to reduce the risk of subsidence, conforms to the natural lordosis of the lumbar spine, and restores the disc height of the operative level. However, these processes do not ensure the implant mechanics will match the patient's anatomy.

Accurate preoperative templating to evaluate the compatibility of the anatomical features of a degenerate lumbar motion segment with the biomechanical parameters of the implant may increase the likelihood of achieving a successful outcome. With this aim in mind we have developed a patientspecific, preoperative templating method using finite element (FE) methods. The objectives of this study were (1) to validate the templating method by comparing the model-predicted ROM with ROM measured from actual postoperative lateral radiographs, (2) to retrospectively determine the ideal implant size, height, configuration, and location to evaluate if the ROM achieved could have been improved, and (3) to evaluate the clinical outcomes relative to the ROM achieved.

\section{MATERIALS AND METHODS}

Seventeen patients, 11 males and 6 females with an average age of 36 years ranging from 21 to 50 (Table 1), were implanted with 22 ProDisc-L TDRs at L4-L5 and or L5-S1 between 2008 and 2015. Single-level surgery was performed on 12 patients, and 2-level surgery on 5 patients. All patients were instructed to restrict bending, twisting, and lifting more than 10 pounds for the first 6 weeks, after which restrictions were lifted and patients were encouraged to increase activity as tolerated.
Computer-aided design models of the 4 ProDisc$\mathrm{L}$ implants were obtained $\left(6^{\circ}\right.$ medium, $6^{\circ}$ large, $11^{\circ}$ medium, $11^{\circ}$ large) from Synthes (West Chester, Pennsylvania) prior to their acquisition by Johnson \& Johnson (Figure 1). The device is composed of 2 metal endplates that are stabilized in the midline of the superior and inferior vertebral endplates and a polyethylene inlay that snap-locks into the inferior endplate and articulates with the superior endplate. While this arrangement approximates the axis of rotation of a natural disc, this implant has a fixed center of rotation, whereas the natural segment's axis of rotation is not fixed but moves in flexion, extension, ${ }^{10,11}$ and lateral bending. ${ }^{12}$

Standing flexion, extension, and neutral radiographs of all segments were obtained at the 6-week follow-up, and again 3, 6, and 12 months after surgery (Figure 2). All patients had radiographs, including flexion and extension films performed 6 weeks postsurgery that were available for analysis. Incomplete sets of radiographs were available at 3 , 6 , and 12 months postsurgery. For this reason, and to eliminate any extraneous, temporal variables, only radiographs performed at 6-week follow up were evaluated. At this stage, patients had recovered from surgery and they had resumed normal activity. Clinical outcome was measured using number rating scale (NRS) and the Oswestry Disability Index (ODI). Pearson correlation coefficients were computed between the measured ROM and the ODI scores reported at 6 weeks follow-up. The postop-

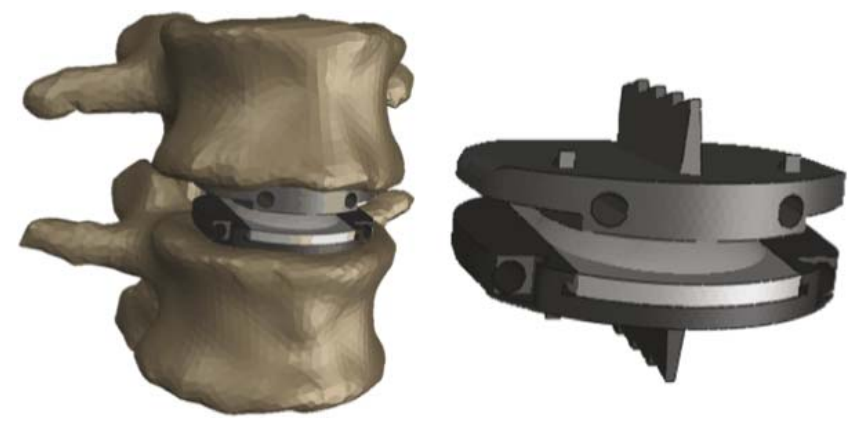

Figure 1. Second generation ProDisc- $L$ total disc replacement device is based on a ball-and-socket concept. The design consists of a superior endplate with a central keel, a high modulus polyethylene inlay, and an inferior endplate with central keel. 

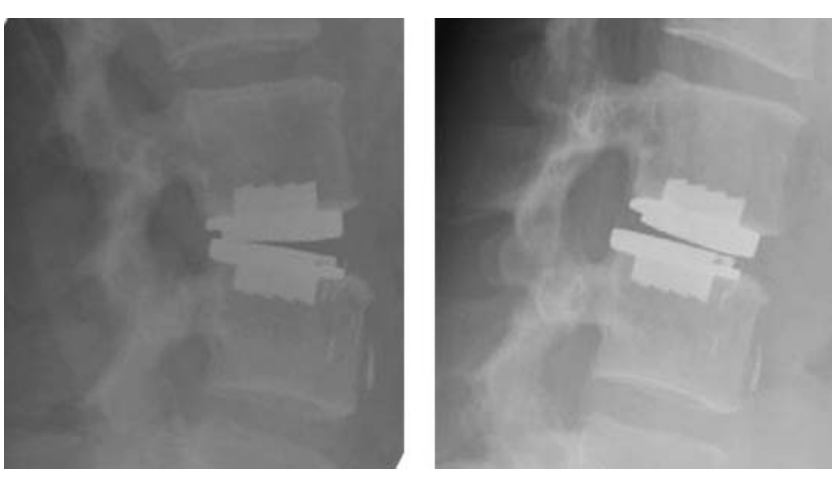

Figure 2. Postoperative follow-up examination at 6 weeks. Standing, lateral radiographs in extension (left) and flexion (right).

erative ROM of the operative level was calculated as the difference between the angular position of the superior endplate in flexion and its angular position in extension as measured from the standing radiographs (Figure 3). This method to calculate postoperative ROM was verified using the technique outlined in Lim et al, ${ }^{13}$ which measured segmental ROM as the change in angle of radiographic landmarks on the TDR implant from flexion to extension. The overlay and the Lim technique were compared using a paired $t$-test.

Three-dimensional FE models of each instrumented segment were created. Vertebral geometries were extracted from preoperative computed tomographic (CT) scans (Figure 4), usually obtained in conjunction with lumbar discography, using Scan IP

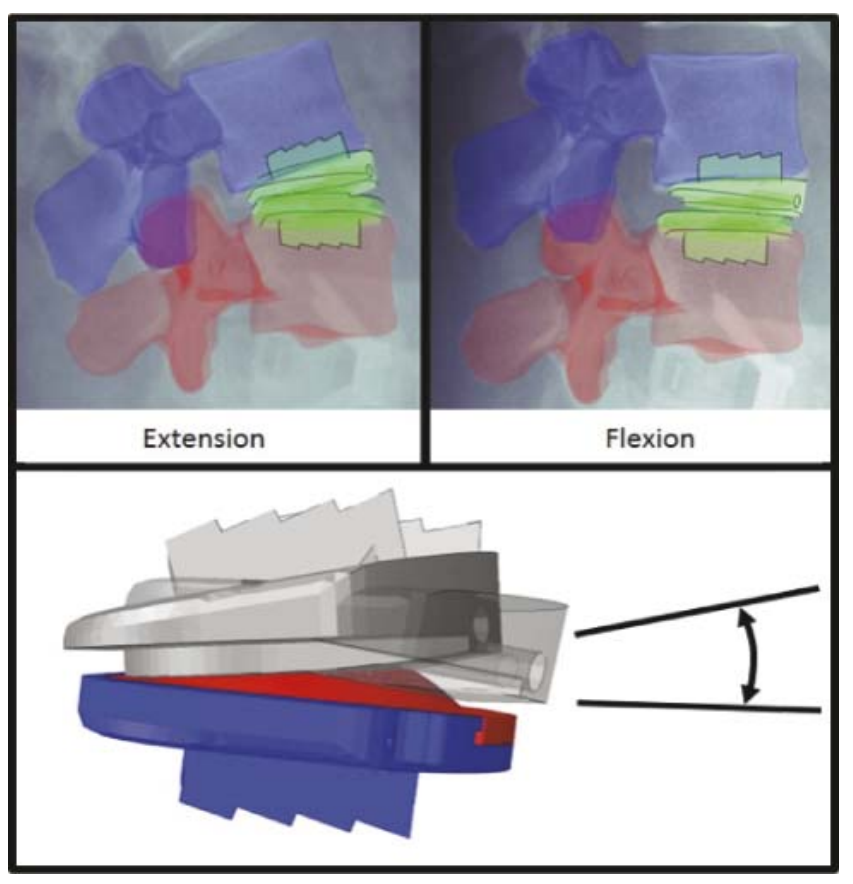

Figure 3. Three-dimensional model overlay with flexion and extension x-ray images. Change in angle measured from resulting 3D model.

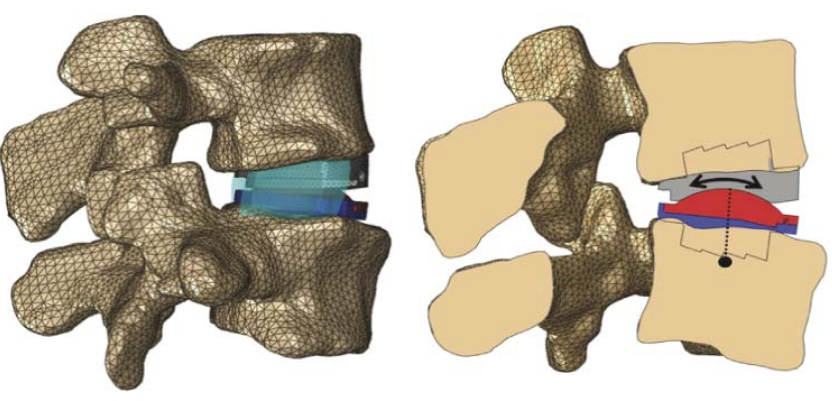

Figure 4. Three-dimensional finite element model aligned to its loaded neutral configuration, includes vertebral bodies, implant in neutral position, lateral annulus, and major elements (left). The rigid body reference node of the superior vertebra (black circle) is located at the center of rotation of the superior vertebra and superior endplate of the implant (right).

(Simpleware, Exeter, UK) and discretized into rigid, tetrahedral elements using Hypermesh (Altair, Troy, Michigan). The average CT image pixel size was $0.31 \mathrm{~mm}$, and the average slice thickness was $1 \mathrm{~mm}$. Rigid, tetrahedral elements modeled the components of the implant, and a perfect bond was assumed between the implant endplates and their adjacent vertebra. A ProDisc-L TDR anterior approach and mobilization of the segment was simulated by removal of the anterior and posterior annulus fibrosus (AF), the entire nucleus pulposus, and the posterior longitudinal ligament. The lateral regions of the AF remained intact and were represented by deformable, first-order, hexahedral elements. A hyperelastic, anisotropic material with circumferential fiber orientation was assigned to the lateral AF. A perfect bond was assumed between the $\mathrm{AF}$ and the adjacent vertebrae. Five major ligaments and capsular structures including the supraspinous, interspinous, and intertransverse ligaments, the facet capsule and the ligamentum flavum were represented as 2-noded, nonlinear connector elements. The anterior longitudinal ligament was not included in the model. A pressure-overclosure relationship of 10.0, which was based on prior computational efficiency studies,${ }^{14}$ was established between the vertebrae, the superior endplate, and the inferior endplate. The inferior vertebral body was fixed in all degrees of freedom at its rigid body reference node.

To simulate flexion and extension, pure moments were applied to the superior vertebra, which ensured articulation between the concave surface of the superior endplate of the implant and the dome of the inlay. In flexion, as the superior endplate rotated around the inlay, the superior vertebra translated anteriorly relative to the inferior vertebra until facet contact occurred. In 


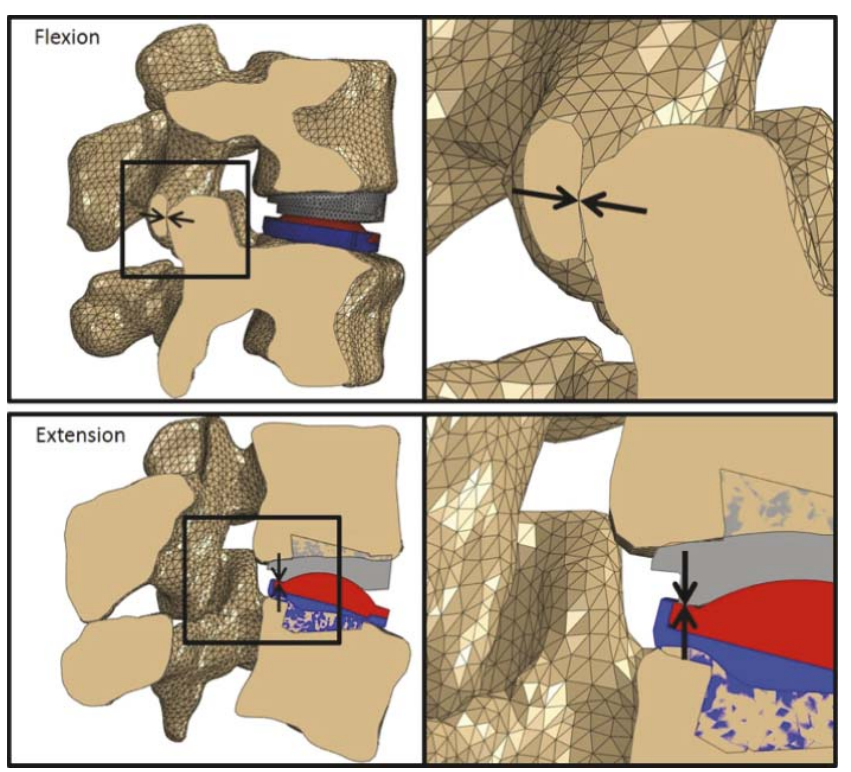

Figure 5. Range of motion evaluation from the templating procedure is typically limited by facet impingement in flexion (above) and implant impingement in extension (below).

extension, the superior vertebra translates posteriorly until the superior and inferior endplates of the implant made contact (Figure 5). Total ROM was calculated by adding the ROM in flexion to that in extension. Initial positions of the vertebrae and the implant components of the model were manually aligned to positions observed in the standing, neutral lateral radiograph using a custom MATLAB script (MathWorks, Natick, Massachusetts), and ROM was calculated in this position. The model was validated by comparing predicted ROM to postoperative ROM in extension, flexion, and the total ROM using a paired $t$-test.

The templating method enables preoperatively determination of the ideal location and configuration of the implant to optimize postoperative ROM. To demonstrate the efficacy of this method, the ideal location and configuration of the implant was retrospectively determined for the current sample by assessing ROM of the motion segment with the implant placed so that the posterior aspect of the inferior implant endplate was at the posterior margin of the inferior vertebra. Both the superior and inferior implants were then moved anteriorly in $0.5-\mathrm{mm}$ increments, reassessing the ROM achieved in each location. This process was repeated for each of the sizes and angles available, which enabled the identification of the ideal implant configuration and optimal location to maximize the ROM that could potentially be

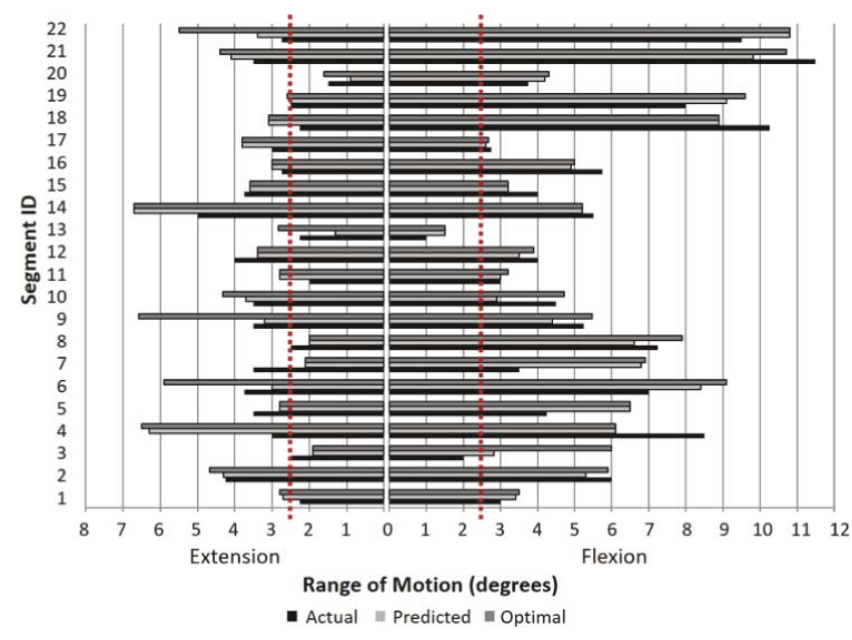

Figure 6. Comparison of actual, predicted, and optimal range of motion (ROM) in flexion (right) and extension (left) for patient cohort. Increased risk of adjacent segment disease can occur if postoperative ROM of at least $5^{\circ}$ is not achieved, indicated here by the region within the dotted lines.

achieved based on vertebral size and facet orientation. The optimal ROM was then compared to the postoperative ROM achieved using a paired $t$ test.

\section{RESULTS}

No significant difference in calculating ROM was evident between the image overlay technique and the measurement method introduced by Lim et $\mathrm{al}^{13}$ $(P=.32)$ Thus, data obtained via the overlay method are presented here (Figure 6).

Model ROM data at the actual implant position were acquired for extension, flexion, and total ROM from the previously described templating procedure. The measured and calculated ROM in flexion, extension, and total motion are summarized in Table 2. No significant difference was evident between the postoperative ROM measured from radiographs and $\mathrm{ROM}$ calculated using the model in flexion $(P=.48)$, in extension $(P=.26)$, and total $\operatorname{ROM}(P=.30)$.

Optimal implant size and placement was determined via the templating method described above for all 22 implanted segments, with the resulting maximum ROM calculated to be significantly greater than the actual ROM $(P=.0005)$ (Table 2 ). Greater ROM could have been achieved in 19 of the 22 segments $(86 \%)$ had the implant been placed in the ideal location or had a different implant been selected and placed in the ideal location. In 8 of 22 levels, ROM could have been improved by more than $15 \%$. While greater total ROM could have been achieved in the majority of implanted seg- 
Table 2. Comparison of actual (ACT), predicted (PRED), and optimal (OPT) range of motion (ROM) data for flexion, extension, and total ROM showing the mean and standard deviation (SD) for the 22 implanted levels. Comparisons of actual versus predicted ROM revealed no significant difference $(P=.26-.48)$, and a significant difference was identified between predicted and optimal ROM $(P=.003-.008)$. Bolded values indicate instrumented levels that could have achieved over $1^{\circ}$ greater total ROM had a preoperative templating process been utilized. Italic indicates the instrumented level that would have achieved less than $5^{\circ}$ of motion even with the optimal implant in the ideal location.

\begin{tabular}{|c|c|c|c|c|c|c|c|c|c|}
\hline \multirow[b]{2}{*}{ ID } & \multicolumn{3}{|c|}{ Extension } & \multicolumn{3}{|c|}{ Flexion } & \multicolumn{3}{|c|}{ Total } \\
\hline & $\mathrm{ACT}$ & PRED & OPT & ACT & PRED & OPT & ACT & PRED & OPT \\
\hline 1 & 2.3 & 2.7 & 2.8 & 3.0 & 3.4 & 3.5 & 5.3 & 6.1 & 6.3 \\
\hline 2 & 4.3 & 4.3 & 4.7 & 6.0 & 5.3 & 5.9 & 10.3 & 9.6 & 10.6 \\
\hline 3 & 2.5 & 1.9 & 1.9 & 2.0 & 2.8 & 6.0 & 4.5 & 4.7 & 7.9 \\
\hline 4 & 3.0 & 6.3 & 6.5 & 8.5 & 6.1 & 6.1 & 11.5 & 12.4 & 12.6 \\
\hline 5 & 3.5 & 2.8 & 2.8 & 4.3 & 6.5 & 6.5 & 7.8 & 9.3 & 9.3 \\
\hline 6 & 3.8 & 3.0 & 5.9 & 7.0 & 8.4 & 9.1 & 10.8 & 11.4 & 15.0 \\
\hline 7 & 3.5 & 2.1 & 2.1 & 3.5 & 6.8 & 6.9 & 7.0 & 8.9 & 9.0 \\
\hline 8 & 2.5 & 2.0 & 2.0 & 7.3 & 6.6 & 7.9 & 9.8 & 8.6 & 9.9 \\
\hline 9 & 3.5 & 3.2 & 6.6 & 5.3 & 4.4 & 5.5 & 8.8 & 7.6 & 12.0 \\
\hline 10 & 3.5 & 3.7 & 4.3 & 4.5 & 2.9 & 4.7 & 8.0 & 6.6 & 9.0 \\
\hline 11 & 2.0 & 2.8 & 2.8 & 3.0 & 3.0 & 3.2 & 5.0 & 5.8 & 6.0 \\
\hline 12 & 4.0 & 3.4 & 3.4 & 4.0 & 3.5 & 3.9 & 8.0 & 6.9 & 7.3 \\
\hline 13 & 2.3 & 1.3 & 2.9 & 1.0 & 1.5 & 1.5 & 3.3 & 2.8 & 4.3 \\
\hline 14 & 5.0 & 6.7 & 6.7 & 5.5 & 5.2 & 5.2 & 10.5 & 11.9 & 11.9 \\
\hline 15 & 3.8 & 3.6 & 3.6 & 4.0 & 3.2 & 3.2 & 7.8 & 6.8 & 6.8 \\
\hline 16 & 2.8 & 3.0 & 3.0 & 5.8 & 4.9 & 5.0 & 8.5 & 7.9 & 8.0 \\
\hline 17 & 3.0 & 3.8 & 3.8 & 2.8 & 2.6 & 2.7 & 5.8 & 6.4 & 6.5 \\
\hline 18 & 2.3 & 3.1 & 3.1 & 10.3 & 8.9 & 8.9 & 12.5 & 12.0 & 12.0 \\
\hline 19 & 2.5 & 2.5 & 2.6 & 8 & 9.1 & 9.6 & 10.5 & 11.6 & 12.2 \\
\hline 20 & 1.5 & 0.9 & 1.6 & 3.8 & 4.2 & 4.3 & 5.3 & 5.1 & 5.9 \\
\hline 21 & 3.5 & 4.1 & 4.4 & 11.5 & 9.8 & 10.7 & 15 & 13.9 & 15.1 \\
\hline 22 & 2.8 & 3.4 & 5.5 & 9.5 & 10.8 & 10.8 & 12.3 & 14.2 & 16.3 \\
\hline Mean & 3.1 & 3.2 & 3.8 & 5.5 & 5.5 & 6.0 & 8.5 & 8.7 & 9.7 \\
\hline SD & 0.8 & 1.3 & 1.6 & 2.7 & 2.6 & 2.6 & 2.9 & 3.1 & 3.2 \\
\hline Maximum & 5.0 & 6.7 & 6.7 & 11.5 & 10.8 & 10.8 & 15.0 & 14.2 & 16.3 \\
\hline Minimum & 1.5 & 0.9 & 1.6 & 1.0 & 1.5 & 1.5 & 3.3 & 2.8 & 4.3 \\
\hline$t$ test & \multicolumn{3}{|c|}{$P=.26$} & \multicolumn{3}{|c|}{$P=.48$} & \multicolumn{3}{|c|}{$P=.30$} \\
\hline$P$ value & \multicolumn{3}{|c|}{$P=.008$} & \multicolumn{3}{|c|}{$P=.003$} & \multicolumn{3}{|c|}{$P=.0005$} \\
\hline
\end{tabular}

ments, in only one were anatomical features identified that limited motion to less than $5^{\circ}$ with the optimal implant placed in the ideal position.

A Pearson correlation coefficient of 0.66 was computed between the predicted ROM and the available ODI scores at 6-week follow-up. Significance of this correlation could not be realized due to a small sample size and missing data.

\section{DISCUSSION}

Acceptance of the validity of computer generated models to clinical applications such as the preoperative assessment of patient suitability, implant selection, and location for lumbar TDR is not universal. One purpose of this study was to validate the model by comparing the predicted ROM to the actual ROM achieved postoperatively. Proper model validation is based on quantitative comparisons between experimental and computational outcomes. ${ }^{15}$ In our study, model ROM predictions were quantitatively compared to actual ROM achieved postoperatively. Model-predicted and actual ROM values were not significantly different and the corresponding percent error in total ROM was $11.7 \%$.

Results of this study were not sensitive to the loading conditions of the models, as flexionextension ROM was predicted based on facet and implant impingement alone. In reality, soft tissue structures such as facet capsules, residual annular tissue, and lumbar musculature also play a role in load sharing and, thus, ROM in the spinal segment during motion. The degree of segmental mobilization performed at the time of surgery, the formation of postoperative scar tissue as well as facet capsule tension or inflammation may limit the motion evident postsurgery and explain discrepancies in the actual and predicted ROM. The 6 weeks postsurgery flexion and extension radiographs were selected to determine the actual ROM achieved as all patients had these films available for comparison and at this point in their recovery there would be little or no effect due to scar tissue formation or facet disease. Currently, load-sharing contributions can be determined in vitro by modeling the torquerotation behavior of the joint. For the purposes of this study, however, the effect of soft tissue 




Figure 7. Facet geometry influences range of motion (ROM) in flexion. The smaller overall size of the vertebrae in combination with more coronally orientated and vertical facet joints in segment 13 is responsible for the small ROM achieved (left). On the contrary, the larger vertebrae, more sagittally orientated and rounded or flatter facet joints in the patient who received segment 14 resulted in a significantly higher ROM and was associated with better clinical outcome (right).

structures on load sharing and ROM were not studied, and only contact between the facets and components of the implant were considered as limiting factors of ROM. While lateral bending and axial rotations influence normal function of an instrumented segment, our prior work revealed that these motions are less dependent on implant position than the anatomical features of the motion segment itself. As a result, motion in these planes was not assessed in this study.

Others have suggested that more diligent and accurate screening methods could boost overall outcomes and persuade healthcare insurers to provide coverage for lumbar TDR. Siepe et al ${ }^{16}$ expands upon prevailing exclusion criteria to suggest that patients with pre-existing degenerative facet joint arthropathies (FJA) should be advised against a TDR procedure. $^{17,18}$ Strube et al ${ }^{19}$ cautions that overdistraction of the facet joint or excessive translation of the superior vertebra can accelerate FJA and lead to negative patient outcomes. Dreischarf et $\mathrm{al}^{20}$ reports that a more posterior placement of a fixed-axis implant, such as the ProDisc-L, increases ROM at the operated level, however, Rohlmann et $\mathrm{al}^{21}$ reported that the most posterior implant placement does not guarantee maximum ROM, and optimal implant placement is patient-specific.

Results of the templating method in the current study elucidated how key anatomical differences influenced ROM. In practice, surgeons can assess facet angles with MRI and CT imaging. However, the facet angles' influence on ROM can be precisely determined with computer modeling and considering the relatively small ROM of a single functional spine unit, changes of a degree or two may be clinically significant. In the case of the implanted segment 13 in our series, a small vertebra with more coronally orientated facet joints and a facet angle of $140^{\circ}$ resulted in limitation of the total ROM to less than $5^{\circ}$ when the optimal implant was placed in the ideal location (Figure 7). This patient reported a postoperative ODI improvement of only 9 points. Conversely, Segment 14 achieved $12^{\circ}$ total ROM and exhibited more sagittally orientated facets with a facet angle of $155^{\circ}$. This patient reported a postoperative ODI improvement of 19 points. While both patients improved, from their preoperative status we believe the clinical and functional outcomes are linked. Overall, had these implanted levels been preoperatively templated, ROM could have been improved by more than $10 \%$ in over half of the cohort $(63 \%)$ and by more than $20 \%$ in 5 cases.

While pre- and postoperative ODI scores were not available for all patients, segmental ROM was well correlated to the ODI scores available, supporting the findings reported by Huang ${ }^{9}$ that indicated greater ROM is related to better patient outcomes, which we expect over time will also be associated with a reduced incidence of ASD.

This investigation is limited in several ways. First, the sample size is small, and 5 patients underwent 2level TDR surgeries. However, the templated and optimal ROM calculated is not influenced by the presence of an adjacent level implant; therefore, we do not believe the inclusion of 2-level cases compromises the findings or conclusions of the study. The acquired data are also inherently limited by the resolution of the radiographs and available CT scans. Lastly, the retrospective study design carries the risk of projection bias and is generally considered a lower level of evidence than prospective study designs. A prospective study design would answer our follow-up and future research question which is, if we can determine the ideal implant size and location, can placement be achieved technically with available intraoperative imaging in the operating room, and does this lead to greater ROM achieved and improved clinical outcomes?

While previous groups have focused on clinical outcomes, ROM and reoperation rates compared to fusion procedures with larger cohorts of patients, ${ }^{4-9}$ this study focused on the evaluation of postopera- 
tive ROM and the identification of anatomical features that influence the ROM achieved and potentially clinical outcomes. Our model enabled the identification of the optimal implant size and placement using computational techniques and those patients whose anatomical features were not compatible with the available implant geometries. Development of accurate, robust patient-specific FE models is time intensive, with approximately 50 explicit evaluations run to determine optimal implant size and position for each operative level. Thus, a cohort size of 22 implanted segments was considered sufficient to achieve the objectives of this study.

The utility and value of the templating method are built on the premise that increased ROM leads to more favorable patient outcomes, but it is important to note that excessive ROM may also contribute to negative outcomes. Capsular tensile forces and facet joint forces are dependent upon implant placement with more posterior positioned implants yielding larger facet joint loads. Misalignment or imbalance in the anterior-posterior implant position can transfer increased loads through the facet joints and accelerate FJA. Consideration of facet joint loads in the templating method may improve patient outcomes and reduce revision surgeries due to FJA. In addition, segments with smaller vertebrae or coronally orientated facet joints can achieve improved ROM by placing a smallest implant more posteriorly on the inferior vertebral endplate. However, the smaller footprint size will reduce endplate coverage, which may lead to increased risks of device migration and subsidence. Increased device ROM may also lead to increased wear and accelerated failure of the bearing surface. These are all factors that warrant further evaluation, and the correlation of the ROM achieved and identification of the optimal implant configuration and location will lead to greater understanding of when and how this technology should be used to maximize patient outcomes and function.

The templating method retrospectively demonstrated that the postoperative ROM achieved could have been improved in $86 \%$ of the cohort had the implant been selected and/or positioned differently. One case was identified where patient size and anatomy was considered to be unsuitable for this TDR implant based on the optimal achievable ROM. Patient-specific templating for lumbar TDR utilizing FE methods may be effective and has the potential to improve patient outcomes. This method is useful in matching patient anatomy to an implant with a fixed axis of rotation. As variation in patient anatomy, including vertebral size and facet orientation, influences ROM, implant manufacturers can also use this tool to modify key design features including implant size, instrumentation to optimize implant positioning, and bearing surface configuration to match a patient's anatomy to its optimal implant configuration.

Future work will focus on building the cohort of patients and to evaluate the differences between implants with a fixed axis of rotation to those with different bearing surfaces, geometries, and biomechanics to identify factors, both implant and patient related, that influence the outcome of TDR surgery.

\section{REFERENCES}

1. Yoshihara H, Yoneoka D. National trends in the surgical treatment for lumbar degenerative disc disease: United States, 2000 to 2009. Spine J. 2015;15(2):265-271.

2. Zeilstra DJ, Miller LE, Block JE. Axial lumbar interbody fusion: a 6-year single-center experience. Clin Interv Aging. 2013;8:1063-1069.

3. Sears WR, Sergides IG, Kamemi N, Smith M, White GJ, Osburg B. Incidence and prevalence of surgery at segments adjacent to a previous posterior lumbar arthrodesis. Spine J. 2011;11(1):11-20.

4. Blumenthal S, McAfee PC, Guyer RD, et al. A prospective, randomized, multicenter food and drug administration investigational device exemptions study of lumbar total disc replacement with the CHARITE artificial disc versus lumbar fusion: part i: evaluation of clinical outcomes. Spine (Phila Pa 1976). 2005;30(14):1565-1575.

5. Guyer RD, McAfee PC, Banco RJ, et al. Prospective, randomized, multicenter Food and Drug Administration investigational device exemption study of lumbar total disc replacement with the CHARITE artificial disc versus lumbar fusion: five-year follow-up. Spine J. 2009;9(5):374-386.

6. Delamarter R, Zigler JE, Balderston RA, Cammisa FP, Goldstein JA, Spivak JM. Prospective, randomized, multicenter Food and Drug Administration investigational device exemption study of the ProDisc-L total disc replacement compared with circumferential arthrodesis for the treatment of two-level lumbar degenerative disc disease. $J$ Bone Joint Surg Am. 2011;93(8):705-715.

7. Gornet MF, Burkus JK, Dryer RF, Peloza JH. Lumbar disc arthroplasty with MAVERICK disc versus stand-alone interbody fusion: a prospective, randomized, controlled, multicenter investigational device exemption trial. Spine (Phila Pa 1976). 2011;36(25):E1600-E1611.

8. Harrop JS, Youssef JA, Maltenfort M, et al. Lumbar adjacent segment degeneration and disease after arthrodesis and total disc arthroplasty. Spine (Phila Pa 1976). 2008;33(15):1701-1707.

9. Huang RC, Tropiano P, Marnay T, Girardi FP, Lim MR, Cammisa FP. Range of motion and adjacent level degeneration after lumbar total disc replacement. Spine J. 2006;6(3):242-247. 
10. Ogston NG, King GJ, Gertzbein SD, Tile M, Kapasouri A, Rubenstein JD. Centrode patterns in the lumbar spine: baseline studies in normal subjects. Spine (Phila Pa 1976). 1986;11(6):591-595.

11. Pearcy MJ, Bogduk N. 1988. Instantaneous axes of rotation of the lumbar intervertebral joints. Spine (Phila $\mathrm{Pa}$ 1976). 1988;13(9):1033-1041.

12. Rousseau MA, Bradford DS, Hadi TM, Pedersen KL, Lotz JC. The instant axis of rotation influences facet forces at L5/S1 during flexion/extension and lateral bending. Euro Spine J. 2006;15(3):299-307.

13. Lim MR, Loder RT, Huang RC, et al. Measurement error of lumbar total disc replacement range of motion. Spine (Phila Pa 1976). 2006;31(10):E291-E297.

14. Deacy JS, Rao M, Smith S, Petrella AJ, Laz PJ, Rullkoetter PJ. Combined rigid-deformable modeling of lumbar spine mechanics. ASME Summer Bioengineering Conference; June 16-19, 2010. https://doi.org/10.1115/ SBC2010-19672.

15. Anderson AE, Ellis BJ, Weiss JA. Verification, validation and sensitivity studies in computational biomechanics. Comput Methods Biomech Biomed Engin. 2007;10(3):171184.

16. Siepe CJ, Heider F, Haas E, et al. Influence of lumbar intervertebral disc degeneration on the outcome of total lumbar disc replacement: a prospective clinical, histological, X-ray and MRI investigation. Euro Spine J. 2012;21(11):2287-2299.

17. Geisler FH, Guyer RD, Blumenthal SL, et al. Patient selection for lumbar arthroplasty and arthrodesis: the effect of revision surgery in a controlled, multicenter, randomized study. J Neurosurg Spine. 2008;8:13-16.

18. Quirno M, Goldstein JA, Bendo JA, Kim Y, Spivak JM. The incidence of potential candidates for total disc replacement among lumbar and cervical fusion patient populations. Asian Spine J. 2011;5(4):213-219.
19. Strube P, Hoff EK, Schmidt H, Dreischarf M, Rohlmann A, Putzier M. Parameters influencing the outcome after total disc replacement at the lumbosacral junction. Part 2: distraction and posterior translation lead to clinical failure after a mean follow-up of 5 years. Euro Spine J. 2013;22(10):22792287.

20. Dreischarf M, Rohlmann A, Zhu R, Schmidt H, Zander $\mathrm{T}$. Is it possible to estimate the compressive force in the lumbar spine from intradiscal pressure measurements? A finite element evaluation. Med Eng Phys. 2013;35(9):1385-1390.

21. Rohlmann A, Mann A, Zander T, Bergmann G. Effect of an artificial disc on lumbar spine biomechanics: a probabilistic finite element study. Euro Spine J. 2009;18(1):89-97.

Disclosures and COI: Part funding for this project was provided through a research grant from the Anschutz Foundation. The authors have no relevant conflicts of interest.

Corresponding Author: Christopher M.J. Cain, Department of Orthopedics, University of Colorado, Denver, 12631 East 17th Ave, Mail Stop B202, Academic Office 1, Room 4611, Aurora, CO 80045. Phone: +1 (303) 724-8936; Fax: +1 (303) 7241593; Email: christopher.cain@ucdenver.edu.

Published 22 February 2019

This manuscript is generously published free of charge by ISASS, the International Society for the Advancement of Spine Surgery. Copyright (c) 2019 ISASS. To see more or order reprints or permissions, see http://ijssurgery.com. 